\title{
Fuzzy implications satisfying convexity relations
}

\author{
Mirko Navara \\ Czech Technical University in Prague, Czech Republic
}

\begin{abstract}
It is an open question whether a non-trivial convex combination of triangular norms (resp. conorms) can be a triangular norm (resp. conorm). We investigate the analogous question for S-implications and R-implications.
\end{abstract}

Keywords: Fuzzy implication, R-implication, Simplication, convex combination, triangular norm, triangular conorm, fuzzy negation.

\section{Fuzzy implications}

The success of fuzzy set theory is mostly based on systems which interpret human knowledge in terms of fuzzy logic. Such systems imitate human way of thinking using fuzzy IF-THEN rules. The interpretation of these rules in terms of fuzzy logical connectives requires fuzzy implications as operations on the continuum of fuzzy truth values, usually the real unit interval $[0,1]$. In order to describe a wide variety of reasoning mechanisms, we need a large repertoire of fuzzy implications $[12,13]$. We refer to $[5,3,4]$ for more arguments and discussion of properties of fuzzy implications. For their general mathematical properties, $[6,10,11,19]$ can be recommended.

We use the following general definition of a fuzzy implication (see $[5,3])$ :

Definition 1 A function $I:[0,1]^{2} \rightarrow[0,1]$ is called a fuzzy implication if it satisfies the following conditions:

(I1) I is decreasing in the first variable,

(I2) $I$ is increasing in the second variable,

(I3) $I(0,0)=I(1,1)=I(0,1)=1, \quad I(1,0)=0$.

Thus we only require that $I$ extends the classical (Boolean) implication and preserves its monotonicity. The conditions of Definition 1 are rather weak, so we shall study more specific fuzzy implications in the sequel.

Let us notice that the class of all fuzzy implications (in the sense of Definition 1) forms a convex set of functions on $[0,1]^{2}$, i.e., if $I_{g}, I_{h}$ are fuzzy implications and $c \in[0,1]$, then $I_{f}:[0,1]^{2} \rightarrow[0,1]$ defined by

$$
I_{f}=c I_{g}+(1-c) I_{h}
$$

is a fuzzy implication, too. We investigate the question which specific classes of fuzzy implications admit convex combinations. In particular, we shall concentrate on so-called S-implications and R-implications and briefly discuss Q-implications. We are interested only in convex combinations (1) which are non-trivial, i.e., $I_{g} \neq I_{h}$ and $c \in(0,1)$, so that $I_{g} \neq I_{f} \neq I_{h}$.

Our observations apply also to fuzzy equivalence operations (=biimplications) derived from these implications.

\section{Basic definitions}

The special classes of implication which are of more interest are usually defined as operations derived from other fuzzy logical operations, triangular norms and conorms and fuzzy implications.

We recall that a triangular norm (a t-norm) $T$ is a commutative, associative, and non-decreasing binary operation defined on the real unit interval $[0,1]$ such that $T(x, 1)=x$ holds for all $x \in[0,1]$ (see $[2,10,24])$. Dually, a triangular conorm (a $t$ conorm) $S$ is a commutative, associative, and nondecreasing binary operation defined on $[0,1]$ such that $S(x, 0)=x$ holds for all $x \in[0,1]$. In this paper, we will be dealing with continuous Archimedean t-norms and t-conorms only. A continuous t-norm $T$, resp. a continuous t-conorm $S$, is called Archimedean if for each $x \in(0,1), T(x, x)<x$, resp. $S(x, x)>x$. A continuous t-norm $T$, resp. a continuous t-conorm $S$, is called strict if it is strictly increasing at every point of the open unit square $(0,1)^{2}$; it is called nilpotent if it is Archimedean, but not strict $[2,10,24]$.

A fuzzy negation is a unary operation $N:[0,1] \rightarrow$ $[0,1]$ which is involutive and non-increasing, i.e., $N(N(x))=x$ and $N(x) \geq N(y)$ for all $x, y \in[0,1]$ such that $x \leq y$. In some papers and monographs (e.g. [7]), more general negations are allowed; then our notion defines so-called strong or involutive fuzzy negation. We shall deal only with involutive fuzzy negations and emphasize this fact explicitly only in formulations of the key problems and results.

We shall need the following lemma which is proved in the Appendix:

Lemma 2 Let $\mathcal{I}$ be an interval and $f, g, h: \mathcal{I} \rightarrow \mathcal{I}$ monotonic bijections. Suppose that there exists $c \in$ $(0,1)$ such that

$$
f=c g+(1-c) h .
$$

and

$$
f^{-1}=c g^{-1}+(1-c) h^{-1} .
$$

Then $f=g=h$. 
The conjunction of assumptions (2), (3) may look strange and applicable rather rarely. However, it is the case if we study convex combinations of functions which are involutive, i.e., inverse to themselves (=satisfying $\forall t \in \mathcal{I}: f(f(t))=t$ ).

Corollary 3 Let $\mathcal{I}$ be an interval and $f, g, h: \mathcal{I} \rightarrow$ $\mathcal{I}$ decreasing involutive bijections. Suppose that there exists $c \in(0,1)$ such that (2) holds. Then $f=g=h$.

This applies to fuzzy negations:

Corollary 4 No fuzzy negation is a non-trivial convex combination of involutive fuzzy negations.

\section{S-implications}

Throughout this section, $I_{S, N}$ (possibly with indices) will be an $S$-implication defined by

$$
I_{S, N}(x, y)=S(N(x), y),
$$

where $S$ is a continuous Archimedean t-conorm and $N$ an (involutive) fuzzy negation. This is a natural fuzzification of the simplest Boolean formula expressing the implication in terms of commutative operations. We have freedom in the choice the tconorm $S$ and negation $N$. We ask whether some choice gives rise to a non-trivial solution to (1).

Problem 5 Find an (involutive) fuzzy negation $N$, different t-conorms $S_{f}, S_{g}, S_{h}$, and a constant $c \in$ $(0,1)$ such that

$$
I_{S_{f}, N}=c I_{S_{g}, N}+(1-c) I_{S_{h}, N}
$$

Condition (4) means that, for all $x, y \in[0,1]$,

$S_{f}(N(x), y)=c S_{g}(N(x), y)+(1-c) S_{h}(N(x), y)$.

Substituting $z=N(x)$, we ask if, for all $z, y \in[0,1]$,

$$
S_{f}(z, y)=c S_{g}(z, y)+(1-c) S_{h}(z, y) .
$$

Thus Problem 5 is equivalent to the following:

Problem 6 Find a t-conorm which is a non-trivial convex combination of two t-conorms.

By duality, this problem is equivalent to an analogous problem, where we consider t-norms instead of t-conorms. This has been published, e.g., in the list of open problems by Alsina, Frank, and Schweizer [1]. Partial results were obtained in $[8,15,20,21,25,26]$. According to $[16,18,22]$, this cannot happen for nilpotent t-norms or t-conorms and some classes of strict t-norms or t-conorms. According to our knowledge, the problem for general strict t-norms and t-conorms is still open.

If we fix the t-conorm and admit different fuzzy negations, we may formulate the following:
Problem 7 Find a continuous Archimedean $t$ conorm $S$, different (involutive) fuzzy negations $N_{f}, N_{g}, N_{h}$, and a constant $c \in(0,1)$ such that

$$
I_{S, N_{f}}=c I_{S, N_{g}}+(1-c) I_{S, N_{h}} .
$$

Condition (5) means that, for all $x, y \in[0,1]$,

$S\left(N_{f}(x), y\right)=c S\left(N_{g}(x), y\right)+(1-c) S\left(N_{h}(x), y\right)$

Substituting $y=0$, we ask if, for all $x \in[0,1]$,

$$
N_{f}(x)=c N_{g}(x)+(1-c) N_{h}(x) .
$$

Thus Corollary 4 gives the negative answer to Problem 7 .

In general, we may choose both t-conorms and fuzzy negations and ask the following:

Problem 8 Find continuous Archimedean $t$ conorms $S_{f}, S_{g}, S_{h}$, (involutive) fuzzy negations $N_{f}, N_{g}, N_{h}$, and a constant $c \in(0,1)$ such that

$$
I_{S_{f}, N_{f}}=c I_{S_{g}, N_{g}}+(1-c) I_{S_{h}, N_{h}} .
$$

and S-implications $I_{S_{f}, N_{f}}, I_{S_{g}, N_{g}}, I_{S_{h}, N_{h}}$ are not equal.

A positive answer to Problem 6 would also answer Problem 8 in the positive. We leave these questions for further study.

\section{R-implications (residuated implications)}

Throughout this section, $I_{T}$ (possibly with an index of $T$ ) will be an $R$-implication (also residuated implication) defined by

$$
I_{T}(x, y)=\max \{z \in[0,1] \mid T(x, z) \leq y\},
$$

where $T$ is a continuous Archimedean t-norm. Rimplications appear naturally in logical foundations of fuzzy set theory (see [7]) and in the algebraic theory of residuated lattices. We ask whether some choice of the t-norm $T$ gives rise to a non-trivial solution to (1):

Problem 9 Find different continuous Archimedean t-norms $T_{f}, T_{g}, T_{h}$ and a constant $c \in(0,1)$ such that

$$
I_{T_{f}}=c I_{T_{g}}+(1-c) I_{T_{h}}
$$

This problem has the negative answer:

Theorem 10 Let $I_{T_{f}}, I_{T_{g}}, I_{T_{h}}$ be $R$-implications induced by continuous Archimedean t-norms $T_{f}, T_{g}, T_{h}$, respectively. Suppose that there exists $c \in(0,1)$ satisfying $(7)$. Then $I_{T_{f}}=I_{T_{g}}=I_{T_{h}}$. 
Proof. Let us suppose that the assumptions of Theorem 10 are satisfied. We assume that there are $x, y \in[0,1]$ such that $I_{T_{f}}(x, y) \neq I_{T_{g}}(x, y)$ and we shall derive a contradiction. Without loss of generality, we assume $I_{T_{f}}(x, y)>I_{T_{g}}(x, y)$. (Otherwise, we interchange $I_{T_{g}}$ and $I_{T_{h}}$ and replace $c$ by $1-c$, then we continue in the same way.)

The case $x \leq y$ leads to $I_{T_{f}}(x, y)=1=I_{T_{g}}(x, y)$, a contradiction. Thus we suppose $x>y$.

We define interval $\mathcal{I}=[y, 1]$ and functions $f, g, h: \mathcal{I} \rightarrow \mathcal{I}$ as follows:

$$
\begin{aligned}
& f(t)=I_{T_{f}}(t, y), \\
& g(t)=I_{T_{g}}(t, y), \\
& h(t)=I_{T_{h}}(t, y) .
\end{aligned}
$$

Functions $f, g, h$ are decreasing bijections. To prove that they are also involutive, notice that, for each $t \in \mathcal{I}, z=I_{T_{f}}(t, y)$ is the unique element of $\mathcal{I}$ such that $T_{f}(t, z)=y$. Similarly, for each $z \in \mathcal{I}$, $t=I_{T_{f}}(z, y)$ is the unique element of $\mathcal{I}$ such that $T_{f}(z, t)=T_{f}(t, z)=y$. Thus

$$
t=I_{T_{f}}(z, y)=I_{T_{f}}\left(I_{T_{f}}(t, y), y\right)=f(f(t)) .
$$

Thus we may apply Corollary 3 to interval $\mathcal{I}$, functions $f, g, h$, and constant $c \in(0,1)$. We obtain $f=g=h$, in particular

$$
\begin{aligned}
& f(t)=g(t)=h(t) \\
& =I_{T_{f}}(t, y)=I_{T_{g}}(t, y)=I_{T_{h}}(t, y)
\end{aligned}
$$

a contradiction.

\section{Q-implications}

Following [11, 19], a Q-implication $I_{S, T, N}$ (possibly with indices) is defined by the formula $I_{S, T, N}(x, y)=S(N(x), T(x, y))$, where $S$ is a tconorm, $T$ is a t-norm, and $N$ is a fuzzy negation. This formula is inspired by implications used in quantum logic $[9,14]$.

Problem 11 Find t-conorms $S_{f}, S_{g}, S_{h}$, t-norms $T_{f}, T_{g}, T_{h}$, (involutive) fuzzy negations $N_{f}, N_{g}, N_{h}$, and a constant $c \in(0,1)$ such that

$$
I_{S_{f}, T_{f}, N_{f}}=c I_{S_{g}, T_{g}, N_{g}}+(1-c) I_{S_{h}, T_{h}, N_{h}} .
$$

and Q-implications $I_{S_{f}, T_{f}, N_{f}}, I_{S_{g}, T_{g}, N_{g}}, I_{S_{h}, T_{h}, N_{h}}$ are not equal.

Defining a Q-implication, the choice of three fuzzy operations gives us much more freedom. This increases the possibility that a non-trivial convex combination can be found among them. We leave this question for further research. Notice that a positive answer to Problem 6 would answer also Problems 5 and 11 in the positive.

\section{Conclusions}

We proved that no non-trivial convex combination of R-implications is an R-implication. Partial results towards this directions were obtained for Simplications. Some instances of this problem are equivalent to the open problem whether a t-norm can be a non-trivial convex combination of t-norms. There is still an open possibility of existence of an easier counterexample for S-implications if the choices of a t-conorm and a fuzzy negations are used simultaneously. Further continuation could be studied for Q-implications, where we have more freedom of choice of a t-conorm, t-norm, and a fuzzy negation.

We hope that our technique of proving the main lemma could find application to other problems in the study of fuzzy logical operations and convex combinations.

\section{Appendix}

Proof of Lemma 2. Let us suppose that the assumptions of Lemma 2 are satisfied. We define the set

$$
\mathcal{U}=\{t \in \mathcal{I} \mid f(t)=g(t)\} .
$$

Then also

$$
\begin{aligned}
\mathcal{U} & =\{t \in \mathcal{I} \mid f(t)=h(t)\} \\
& =\{t \in \mathcal{I} \mid g(t)=h(t)\} .
\end{aligned}
$$

We assume that $\mathcal{U} \neq \mathcal{I}$ and we shall derive a contradiction.

We find an $x \in \mathcal{I} \backslash \mathcal{U}$. It follows from the continuity of $f, g, h$ and from the definition of $\mathcal{U}$ (by an equation) that $\mathcal{U}$ is closed. Thus the set

$$
\{t \in \mathcal{U} \mid t<x\}
$$

has a greatest element; we denote it by

$$
u=\max \{t \in \mathcal{U} \mid t<x\} .
$$

Obviously $u \neq x$. The restrictions of $f, g$ to the interval $(u, x]$ satisfy either

$$
g \uparrow_{(u, x]}<f \uparrow_{(u, x]}
$$

or

$$
g \uparrow_{(u, x]}>f \uparrow_{(u, x]} .
$$

Replacing $g$ by $h$, we obtain the reverse ordering. We assume the former case, thus

$$
g \uparrow_{(u, x]}<f \uparrow_{(u, x]}<h \uparrow_{(u, x]} .
$$

(See Figure 1.) We may use this assumption without loss of generality; in the other case, we interchange $g$ and $h$ and replace $c$ by $1-c$, then we continue as follows.

The convexity condition (2) allows us to quantify the latter inequality:

$$
\begin{aligned}
f(t) & =c g(t)+(1-c) h(t), \\
c(f(t)-g(t)) & =(1-c)(h(t)-f(t)) .
\end{aligned}
$$




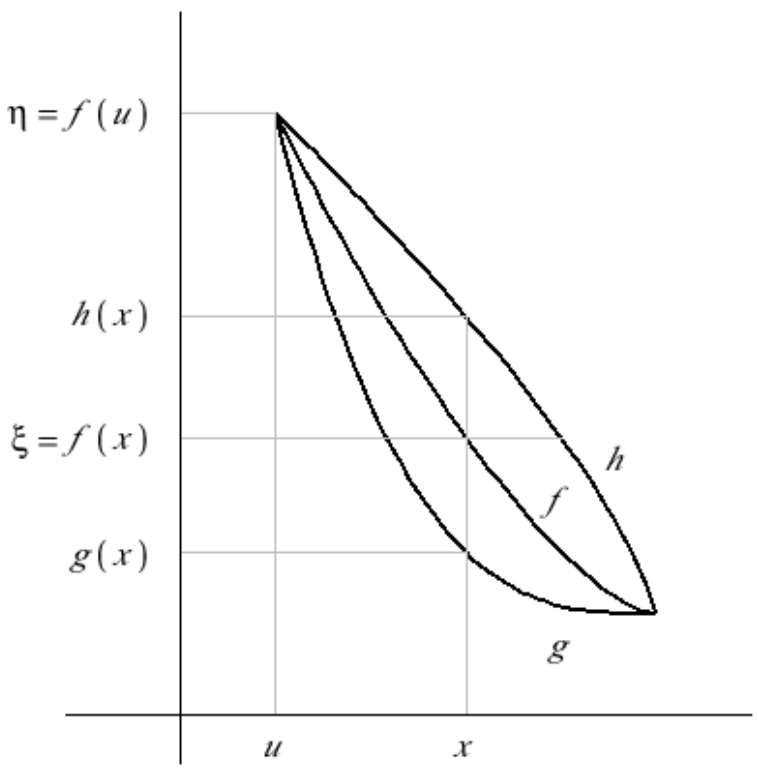

Figure 1: Test of the (im-)possibility of satisfaction of the same convexity relations for the functions and their inverses.

All these functions are continuous, thus we may integrate the latter formula and obtain

$$
\begin{aligned}
& c \underbrace{\int_{u}^{x}(f(t)-g(t)) \mathrm{d} t}_{G} \\
& =(1-c) \underbrace{\int_{u}^{x}(h(t)-f(t)) \mathrm{d} t}_{H},
\end{aligned}
$$

Using the notation

$$
\begin{aligned}
& G=\int_{u}^{x}(f(t)-g(t)) \mathrm{d} t, \\
& H=\int_{u}^{x}(h(t)-f(t)) \mathrm{d} t
\end{aligned}
$$

(see Figure 2), we may write (9) in a simplified form

$$
c G=(1-c) H
$$

Now we shall repeat the above procedure for the inverse functions $f^{-1}, g^{-1}, h^{-1}$ with arguments within the interval swith bounds

$$
\begin{aligned}
& \eta=f^{-1}(u)=g^{-1}(u)=h^{-1}(u), \\
& \xi=f^{-1}(x)
\end{aligned}
$$

(see Figure 3).

According to our assumptions,

$$
g^{-1} \uparrow_{[\xi, \eta)}<f^{-1} \uparrow_{[\xi, \eta)} \mid<h^{-1} \uparrow_{[\xi, \eta)}
$$

and this inequality can be again quantified using (2) for all $\tau \in[\xi, \eta)$ :

$$
\begin{aligned}
f^{-1}(\tau) & =c g^{-1}(\tau)+(1-c) h^{-1}(\tau), \\
c\left(f^{-1}(\tau)-g^{-1}(\tau)\right) & =(1-c)\left(h^{-1}(\tau)-f^{-1}(\tau)\right) .
\end{aligned}
$$

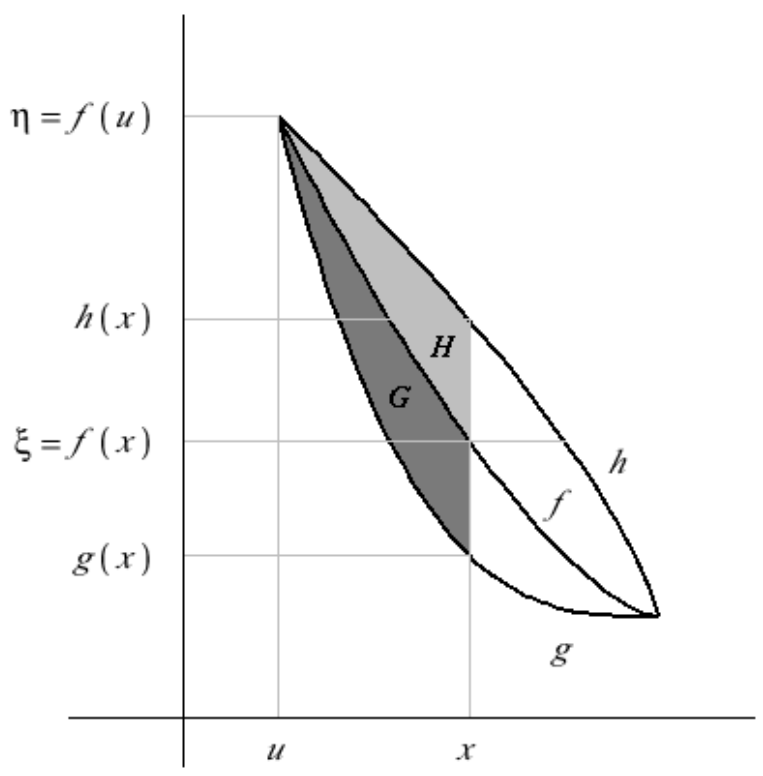

Figure 2: Integrals of differences of functions.

Integrating the latter equality, we obtain

$$
\begin{aligned}
& c \underbrace{\int_{\xi}^{\eta}\left(f^{-1}(\tau)-g^{-1}(\tau)\right) \mathrm{d} \tau}_{G^{\prime}} \\
& =(1-c) \underbrace{\int_{\xi}^{\eta}\left(h^{-1}(\tau)-f^{-1}(\tau)\right) \mathrm{d} \tau}_{H^{\prime}},
\end{aligned}
$$

Using the notation

$$
\begin{aligned}
G^{\prime} & =\int_{\xi}^{\eta}\left(f^{-1}(\tau)-g^{-1}(\tau)\right) \mathrm{d} \tau, \\
H^{\prime} & =\int_{\xi}^{\eta}\left(h^{-1}(\tau)-f^{-1}(\tau)\right) \mathrm{d} \tau
\end{aligned}
$$

(see Figure 3), we may write (12) in a simplified form

$$
c G^{\prime}=(1-c) H^{\prime},
$$

The integral $G$ from (10) can be split (see Figure 2)

$$
\begin{aligned}
G & =\int_{u}^{x}(f(t)-g(t)) \mathrm{d} t \\
& =\underbrace{\int_{\xi}^{\eta}\left(f^{-1}(\tau)-g^{-1}(\tau)\right) \mathrm{d} \tau}_{G^{\prime}} \\
& +\underbrace{\int_{g(x)}^{\xi}\left(x-g^{-1}(\tau)\right) \mathrm{d} \tau}_{D} \\
& =G^{\prime}+D,
\end{aligned}
$$

where

$$
D=\int_{g(x)}^{\xi}\left(x-g^{-1}(\tau)\right) \mathrm{d} \tau>0
$$




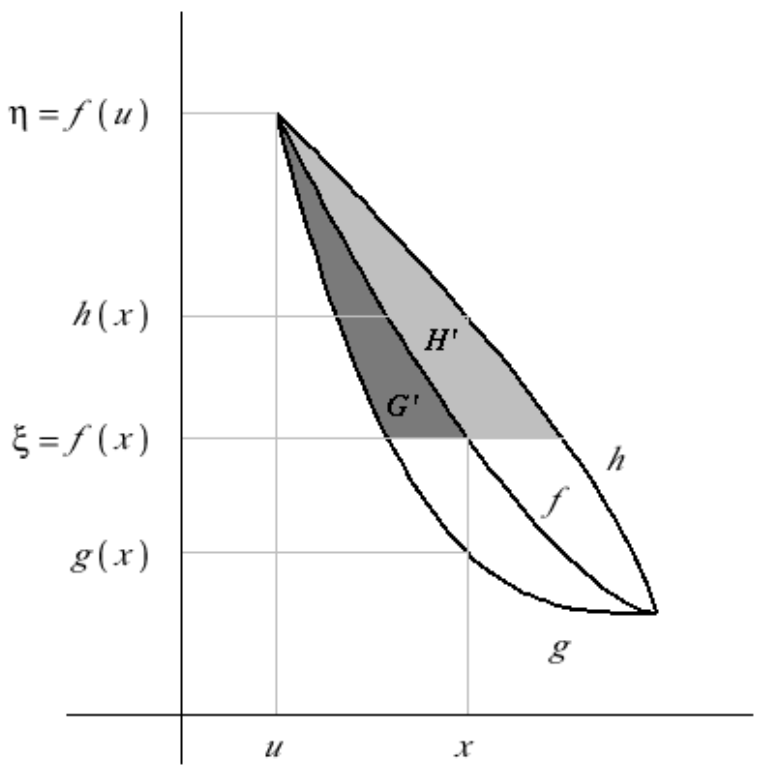

Figure 3: Integrals of differences of inverse functions.

(See Figure 4.)

Similarly for $H^{\prime}$ :

$$
\begin{aligned}
H^{\prime} & =\int_{\xi}^{\eta}\left(h^{-1}(\tau)-f^{-1}(\tau)\right) \mathrm{d} \tau \\
& =\underbrace{\int_{u}^{x}(h(t)-f(t)) \mathrm{d} t}_{H} \\
& +\underbrace{\int_{x}^{h^{-1}(\xi)}(h(t)-\xi) \mathrm{d} t}_{D^{\prime}} \\
& =H+D^{\prime},
\end{aligned}
$$

where

$$
D^{\prime}=\int_{x}^{h^{-1}(\xi)}(h(t)-\xi) \mathrm{d} t>0 .
$$

Combining (11), (13), we obtain

$$
\begin{aligned}
c G^{\prime} & =(1-c) H^{\prime}=(1-c)\left(H+D^{\prime}\right) \\
& >(1-c) H=c G=c\left(G^{\prime}+D\right) \\
& >c G^{\prime},
\end{aligned}
$$

a contradiction. The proof is finished.

Acknowledgement 12 This research was supported by the Czech Technical University in Prague under project $S G S 12 / 187 / O H K 3 / 3 T / 13$.

\section{References}

[1] C. Alsina, M. J. Frank, and B. Schweizer: Problems on associative functions. Aequationes Mathematicae, 66(1-2):128-140, 2003.

[2] C. Alsina, M. J. Frank, and B. Schweizer: Associative Functions: Triangular Norms and Copulas. World Scientific, Singapore, 2006.

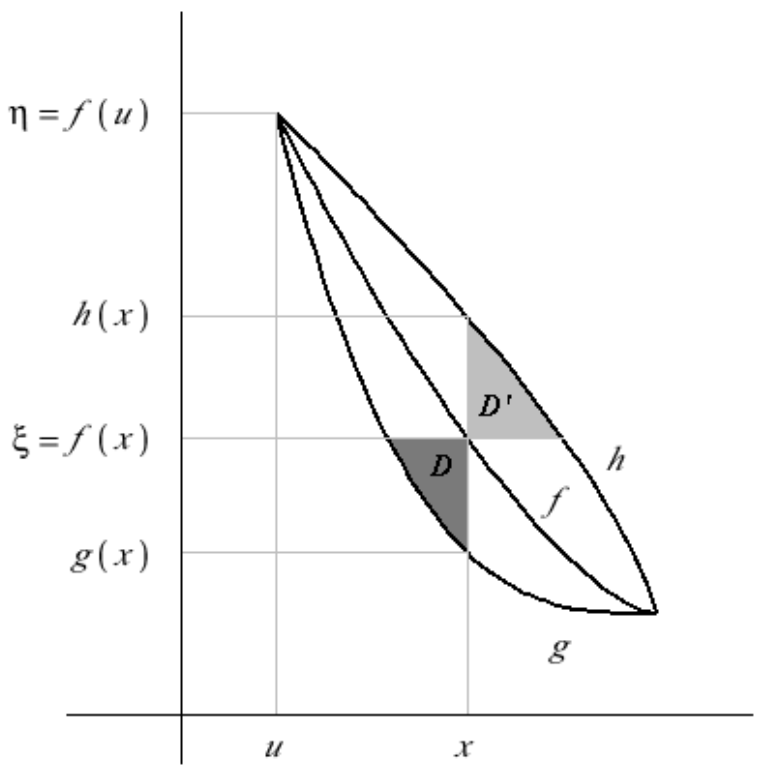

Figure 4: Differences of integrals.

[3] M. Baczyński and B. Jayaram: Fuzzy implications. (Studies in Fuzziness and Soft Computing, Vol. 231), Springer, Berlin, 2008.

[4] M. Baczyński and B. Jayaram: (S,N)- and Rimplications: A state-of-the-art survey. Fuzzy Sets and Systems, 159:1836-1859, 2008.

[5] F. Qin, M. Baczyński, and A. Xie: Distributive equation of implications based on continuous triangular norms. EUSFLAT-LFA 2011, Aixles-Bains, France, 246-253, 2011.

[6] S. Gottwald: A Treatise on Many-Valued Logic. Research Studies Press, Baldock, 2001.

[7] P. Hájek: Metamathematics of Fuzzy Logic. Kluwer Academic Publishers, Dordrecht, 1998.

[8] S. Jenei: On the convex combination of leftcontinuous t-norms. Aequationes Mathematicae, 72(1-2):47-59, 2006.

[9] G. Kalmbach: Orthomodular Lattices. Academic Press, London, 1983.

[10] E. P. Klement, R. Mesiar, and E. Pap: Triangular Norms, vol. 8 of Trends in Logic. Kluwer Academic Publishers, Dordrecht, Netherlands, 2000.

[11] G.J. Klir and B. Yuan: Fuzzy Sets and Fuzzy Logic. Theory and Applications. Prentice-Hall, 1995.

[12] S. Massanet and J. Torrens: A new method of generating fuzzy implications from given ones. EUSFLAT-LFA 2011, Aix-les-Bains, France, 215-222, 2011.

[13] S. Massanet and J. Torrens: On a new class of fuzzy implications: $h$-implications and generalizations. Information Sciences, 181(11):2111 $-2127,2011$.

[14] N.D. Megill and M. Pavičić: Orthomodular lattices and a quantum algebra. International Journal of Theoretical Physics $\mathbf{4 0}$ 
(2001), 1387-1410.

[15] R. Mesiar and A. Mesiarová-Zemánková: Convex combinations of continuous t-norms with the same diagonal function. Nonlinear Analysis: Theory, Methods \& Applications, 69(9):2851-2856, 2008.

[16] M. Navara and M. Petrík: Two methods of reconstruction of generators of continuous tnorms. 12th International Conference Information Processing and Management of Uncertainty in Knowledge-Based Systems, Málaga, Spain, 2008, 1016-1021.

[17] M. Navara, M. Petrík, and P. Sarkoci: Convex combinations of triangular norms. In: U. Bodenhofer, B. De Baets, E.P. Klement, and S. Saminger-Platz (eds.), 30th Linz Seminar on Fuzzy Set Theory: The Legacy of 30 Seminars - Where Do We Stand and Where Do We Go?, Johannes Kepler University, Linz, Austria, 2009, 85-87.

[18] M. Navara, M. Petrík, and P. Sarkoci: Explicit formulas for generators of triangular norms. Publ. Math. Debrecen, 77:171-191, 2010.

[19] H. T. Nguyen and E. Walker: A First Course in Fuzzy Logic. Chapman \& Hall/CRC, Boca Raton, 2nd edition, 2000.

[20] Y. Ouyang and J. Fang: Some observations about the convex combinations of continuous triangular norms. Nonlinear Analysis: Theory, Methods \& Applications, 68(11):33823387, 2008.

[21] Y. Ouyang, J. Fang, and G. Li: On the convex combination of $T_{D}$ and continuous triangular norms. Information Sciences, 177(14):29452953, 2007.

[22] M. Petrík: Convex combinations of strict t-norms. Soft Computing - A Fusion of Foundations, Methodologies and Applications, 14:1053-1057, 2010. DOI: 10.1007/s00500-0090484-3

[23] M. Petrík and P. Sarkoci: Convex combinations of nilpotent triangular norms. Journal of Mathematical Analysis and Applications, 350:271-275, 2009. DOI: 10.1016/j.jmaa.2008.09.060

[24] B. Schweizer and A. Sklar: Probabilistic Metric Spaces. North-Holland, Amsterdam 1983; 2nd edition: Dover Publications, Mineola, NY, 2006.

[25] M. S. Tomás: Sobre algunas medias de funciones asociativas. Stochastica, XI(1):25-34, 1987.

[26] T. Vetterlein: Regular left-continuous t-norms. Semigroup Forum, 77(3):339-379, 2008. 